\title{
Can Selfish Symbioses Effect Higher-level Selection?
}

\author{
Richard A. Watson, Niclas Palmius, Rob Mills, Simon Powers, Alexandra Penn. \\ Natural Systems group, University of Southampton, U.K. \\ raw@ecs.soton.ac.uk
}

\begin{abstract}
The role of symbiosis in macro-evolution is poorly understood. On the one hand, symbiosis seems to be a perfectly normal manifestation of individual selection, on the other hand, in some of the major transitions in evolution it seems to be implicated in the creation of new higher-level units of selection. Here we present a model of individual selection for symbiotic relationships where individuals can genetically specify traits which partially control which other species they associate with - i.e. they can evolve species-specific grouping. We find that when the genetic evolution of symbiotic relationships occurs slowly compared to ecological population dynamics, symbioses form which canalise the combinations of species that commonly occur at local ESSs into new units of selection. Thus even though symbioses will only evolve if they are beneficial to the individual, we find that the symbiotic groups that form are selectively significant and result in combinations of species that are more cooperative than would be possible under individual selection. These findings thus provide a systematic mechanism for creating significant higher-level selective units from individual selection, and support the notion of a significant and systematic role of symbiosis in macro-evolution.
\end{abstract}

\section{Introduction: Can individual selection create higher-level selection?}

Symbiotic relationships in general are ubiquitous and uncontroversial, but the role of symbiosis in macro-evolutionary processes such as the major evolutionary transitions (1) and symbiogenesis (the creation of new species through symbiosis) (2), is poorly understood. Clearly, the evolution of symbiotic relationships may change the effective selection pressures on individuals in complex ways - but can they enable higher-level selection? When the fitness of individuals is context sensitive (i.e. under frequency dependent selection) grouping individuals together in small groups can change the average selection pressure on cooperative traits by altering the variance in contexts $(3,4)$. This effect is stronger when group membership is assortative on behavioural traits (5). In most models, however, the existence of groups is presupposed and accordingly any group selection effect observed is unsurprising in the sense that it is fully explained by changes in individual selection given the context of these groups. In contrast, we are interested in scenarios where individually selected traits affect the strength of group selection or create group selection de novo (6). For example, related work addresses the evolution of individually specified traits that affect group size $(7,8)$, or the evolution of markers that influence behavioural grouping (9). Here we address a multi-species scenario where species can evolve symbiotic relationships that allow explicit control over whether they group and who they group with.

Symbiosis, the living together of different species, implies that one species 'seeks out' another, actively controlling (to a limited extent) the species composition of its environmental context. When organisms create their own environments a complex 
dynamic is created between the traits they evolve that affect their symbiotic relationships, and the 'ordinary traits' (traits that do not affect symbioses) they evolve given the context they have created for themselves. Our research question concerns whether it is possible for an individual to evolve symbiotic relationships that cause it to create a significant higherlevel unit of selection. This might seem to be a logical impossibility because for a higherlevel unit of selection to be significant one would ordinarily assert that it must oppose individual selection. And, if a group opposes individual selection then a defector or selfish individual that exploits the group will be fit and take over. Of course, group selection that acts in alignment with individual selection is possible - e.g. individual selection may cause a mixed population to reach some evolutionarily stable strategy (ESS) (10) and group selection that acts in alignment with individual selection might cause a population to reach this ESS more quickly, but it cannot cause it to go somewhere other than the local ESS. But we show this conclusion is too hasty. We show that in cases where group selection acts in alignment with individual selection it can alter evolutionary outcomes. This requires that we consider a different type of evolutionary game, however.

The literature on group selection is largely preoccupied with the prisoners' dilemma (11) - a game that has only one ESS (10) - 'Defect'. Although a group of cooperative individuals is collectively fitter than a group of defectors, the cooperative group can never be stable given that the payoff for Defect is higher than the payoff for Cooperate when playing against other cooperators. Thus if groups are imposed Cooperate:Cooperate will beat Defect:Defect but it is not possible that a Cooperate:Cooperate group can be maintained by individual selection. In contrast, a game that has more than one ESS is a different matter. A coordination game of two strategies, for example, has two ESSs, let's call them A-A and B-B, and these ESSs may have different overall utility, let's say that an A-A group beats a B-B group. But the difference is that in a game that has multiple ESSs, each ESS can be supported by individual selection (there is no 'cheat' strategy that can invade either ESS) and this means that the two groups need not be externally imposed in order to be stable. Nonetheless, the evolutionary outcome can be significantly different from the outcome of individual selection without grouping. For example, with no grouping, if the utility of A-A is only slightly higher than B-B, then the population will reach the ESS that is closest to the initial conditions - for example, if $\mathrm{B}$ has a significant majority this will be the B-B ESS. But with grouping, the A-A ESS can be reached even if B has a significant majority because when A's interact disproportionately with other A's they are fitter than B's. In the models that follow we will show that individual selection causes groups to form that represent combinations of species from different ESSs and thus allows the highest utility ESS to be found.

We intend our model to represent the evolution of symbiotic relationships between species, not just assortativity of behaviours within a single species. Thus we permit competition between heterogeneous groups (e.g. $\mathrm{AB}$ vs $\mathrm{CD}$, where $\mathrm{A}$ and $\mathrm{B}$ are behaviours provided by unrelated species) rather than homogeneous groups (e.g. AA vs $\mathrm{BB}$ ) as would be more conventional in a single-species assortative grouping model (where relatedness and inclusive fitness concepts straightforwardly apply) (12). By using a polyspecies model we can show that the process we model significantly increases the likelihood of reaching a higher-utility ESS even in cases where the basin of attraction for high-utility ESSs is initially very small (13). Note that we do not change the interaction coefficients between species but only change the co-location or interaction probability of species. A species might thus change its fitness by increasing the probability of interacting with another (which is what we mean be a symbiosis) but it cannot change its intrinsic fitness dependency on that species (as might be part of a more general model of 
coevolution - 4,14,15). There are clearly many ways in which organisms can change interaction probabilities with other organisms either subtly or radically (16).

\section{An ecosystem model with evolved symbioses}

Our abstract model of an ecosystem contains $2 N$ species, each of which contains $P$ individuals. The fitness of each individual in each species will depend on the other species present in its local environmental context. A separation of timescales is crucial in this model (15): On the (fast) ecological dynamics timescale species densities within an environmental context change and quickly reach equilibrium, but on this timescale genetic changes are assumed to be negligible. At a much slower genetic evolution timescale, genetic changes that alter symbiotic relationships are significant. The genotype of an individual specifies partnerships with the other $2 N-1$ species that can partially (or completely) determine the combination of species it appears with in the environmental context. We assume that the initial composition of the local environment contains a random combination of species, but for the scenarios we investigate the ecological dynamics have only stable attractors, so the composition of the ecology quickly equilibrates to a subset of species that are stable. Although the frequency of a species may go to zero in a particular ecological context, in other contexts it will persist (i.e. no species are lost). Different individuals are evaluated in the environmental context for some time, and at the end of each period we turn attention to a new randomly initialised ecological context. Ours is therefore not an explicitly spatial model since we have no need to model different environmental contexts simultaneously.

We choose a very simple representation of the local environmental context - a binary vector representing which species are present in non-zero frequency. We suppose that each position in the vector is a 'niche' that may be occupied by one of two possible species that are mutually exclusive, such that some species cannot coexist in the same ecological context. For example, in a forest where deciduous and coniferous trees are competing, patches of the forest may, in simplistic terms, contain either one or the other but not both simultaneously, and simultaneously a patch may contain one species of ant or another but not both, and moreover, the type of tree present may influence which type of ant is fittest., and vice versa. An $N$-bit vector thus indicates which $N$ species, of the possible $2 N$, are present in the environmental context. A species, '------0---', indicates which type it is (e.g. ' 0 ') and which environmental niche in the environmental context it occupies (e.g. $6^{\text {th }}$ ). This choice of representation has some properties that are required and some that are merely convenient. It is necessary for our purposes that not all species are present in all environmental contexts - otherwise, genetically specifying a symbiotic partnership would be redundant. It is also necessary that there are many different possibilities for the species composition in an environmental context - so the number of species present in any one environment should be large and many combinations of species should be allowed. The fact that species are arranged in mutually exclusive pairs is a contrivance for convenience: having all environmental states contain exactly $N$ species allows us to define the environmental state and as $N$-dimensional space and to define fitness interactions between species using an energy function discussed below. And the fact that environmental states are defined using a binary 'present or not' representation rather than a continuous species density model is again a convenience - a continuous model would be interesting to investigate in future.

Each individual in the ecosystem has a fitness that is a function of the other species present in the current environmental context. In principle, this requires an environmentally sensitive fitness function for each species and the resultant ecological dynamics could be arbitrarily complex in general. In the experiments that follow we restrict our attention to 
ecosystems with simple monotone dynamics and point attractors. Such dynamics can be modelled using an 'energy function' $(17,18)$ over environmental states, $e(E)$, such that the fitness of an individual of species, s, given an environmental context, $E$, is determined by the change in energy, $\Delta e(E, s)$ produced by adding $s$ to $E$. That is, the fitness of an individual of species $s$ in context $E$ is, fitness $(s, E)=\Delta e(E, s)=e(E+s)-e(E)$, where ' $E+s$ ' is the environmental state $E$ modified by adding species $s$. (Dynamical systems theory would normally minimise energy, but for familiarity we let positive $\Delta \mathrm{e}$ correspond to positive fitness such that selection tends to increase e).

Each individual has a genotype that defines which other species it forms groups with (see Figure 1). This genotype is simply a binary vector length $2 N$ defining which of $N$ possible ' 0 ' species it groups with followed by which of $N$ possible ' 1 ' species it groups with. Binary relationships of this form are somewhat crude perhaps, but although the partnerships of any one individual are binary, the evolved associations of the species as a whole, as represented by the frequencies of partnerships in the population of individuals for that species, is a continuous variable (to the resolution of 1/population-size). We use the term 'association' to refer to this population-level inter-species average and reserve the word 'partnership' for the binary relationships specified by the genotype of an individual. The meaning of the binary partnership vector for an individual is simply that its fitness, already a function of the environmental context, is modified by the inclusion of its symbiotic partners into that context. Specifically, the fitness of an individual genotype, $g$, belonging to species, $s$, given a context, $E$, is defined as fitness $(g, E)=\Delta e(E, s+S)=$ $e(E+s+S)-e(E)$, where $S$ is the set of species that $g$ specifies as partners.

Using the components introduced above, illustrated in Figure 1, our model operates as defined in Figure 2.

A species, $s:-------0--$

May contain an individual genotype: $<0001100100,0100000010>^{\#}$

This example individual specifies partnerships with the following 5 species:

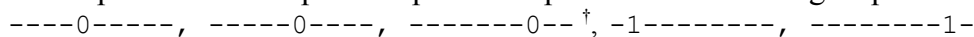

So, if this individual is placed into an environmental context, it and these partner species will be present: i.e. $\mathrm{s}+\mathrm{S}=-1--00-01-$.

For example, if this individual is placed into $\mathrm{E}=1000100000$, with $\mathrm{e}(\mathrm{E})=\alpha$.

It will create $\mathrm{E}+\mathrm{S}+\mathrm{s}=1100000010$, with $\mathrm{e}(\mathrm{E}+\mathrm{S}+\mathrm{s})=\beta$.

And it will receive a fitness of $\Delta \mathrm{e}(\mathrm{E}, \mathrm{S}+\mathrm{s})=\mathrm{e}(\mathrm{E}+\mathrm{S}+\mathrm{s})-\mathrm{e}(\mathrm{E})=\beta-\alpha$.

Figure 1: An individual, its partners and its fitness in an environmental context. ( ${ }^{\dagger}$ For implementational convenience, each individual specifies a partnership with itself. ${ }^{\#}$ A comma indicates separation of 0-partnerships from 1-partnerships.)

\section{A poly-ESS ecological dynamics}

We define the energy of an environmental state, $E$, as a sum over $B$ copies of the subfunction, $f$, applied to disjoint subsets of species as follows:

$e(E)=\sum_{b=0}^{B-1} f\left(\mathrm{~s}_{\mathrm{bk}+1}, \mathrm{~s}_{\mathrm{bk}+2}, \ldots \mathrm{s}_{(\mathrm{b}+1) \mathrm{k}-1}\right) \quad$ where $\quad f(G)= \begin{cases}2, & \text { if } G=N, \\ \max \left(\frac{1}{1+G}, \frac{1}{1+(N-G)}\right) & \text { otherwise. }\end{cases}$

where $B$ is the number of sub-functions, and $k=N / B$ is the number of species in each subfunction. For convenience $f$ is defined as a function of $G$, the number of 1 -species in the subset of species. $f$, defines a simple 'U-shaped' energy function with local optima at all0 s and all-1s, but all-1s has higher energy than all-0s. Concatenating $B$ of these U-shaped sub-functions creates a poly-attractor system. Five subsystems with two attractors each, as 


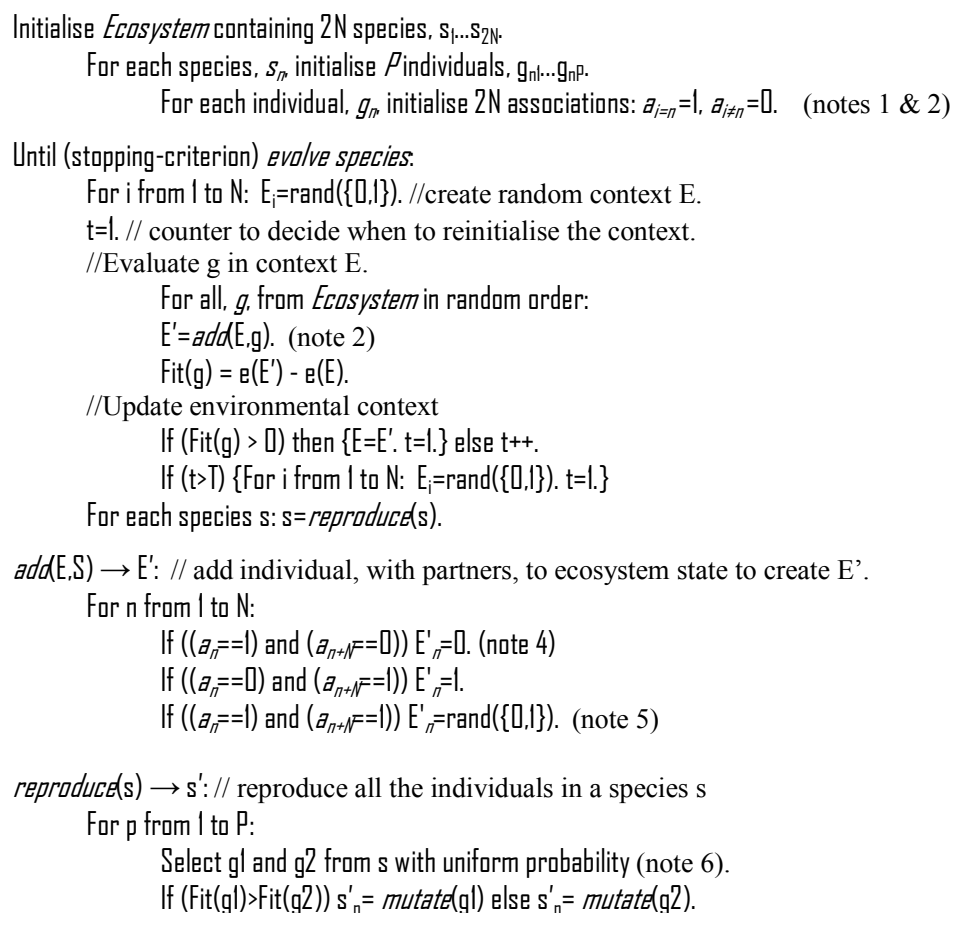

Figure 2: Model details. 1) Initially, each species associates with itself only. 2) Associating with self makes implementation of $\mathrm{E}+\mathrm{S}+\mathrm{s}$ identical to $\mathrm{E}+\mathrm{S}$. 3) This insertion of a species into the ecological state is cumbersome because there are $2 N$ species that fit into $N$ niches. 5) If an individual associates with ' 1 'species and ' 0 '-species that are mutually exclusive (i.e. occupy the same niche) - then either species is added to $E$ with equal probability. 6) Individuals specifying deleterious partnerships (negative fitness) have probability 0 of reproducing, but it is important that individuals specifying neutral partnerships have nonzero probability to reproduce. For the following experiments, $N=50, P=100$, and $T=5 N$ was sufficient to ensure an environmental context found a local ESS before being reinitialised. Mutation is single bit-flip.

used in the following experiments, creates a system with $2^{5}=32$ point attractors (local optima in the energy function, corresponding to ESSs with $N$ species each). For $N=25$, $B=5, \quad k=10, \quad$ a local attractor under individual selection is: 111111111111111111000000000011111111110000000000 . The attractor with the globally-maximal $e$-value is simply the concatenation of the superior solution to each sub-system, i.e. 111111111111111111111111111111111111111111111 . However, which of the two-possible local 'sub-attractors' for each sub-system (e.g. ...1111111111... or ...0000000000...) will be found depends (under individual selection) on whether the initial environmental conditions have type-0s or type-1s in the majority. The all-type-1 attractor for each sub-system is thus found with probability 0.5 from a random initial condition and the probability of finding the global-maximal energy attractor is $0.5^{B}=1 / 32$. (This poly-attractor system is identical to a building-block function used in (19) to show sexual recombination permits selection on subfunctions if genetic linkage is 'tight' - but here we evolve useful linkages.)

\section{Results and Discussion}

Figure 3 (left) shows that under individual selection (before associations are evolved) different attractors are found (categorised by $G$ ). The globally-maximal energy attractor is 
not found in any of the 16 samples depicted. Figure 3 (right) shows that after associations have evolved the globally optimal attractor is being reached in every instance of the local ecological dynamics, regardless of the random initial conditions of the environmental context. Thus the basin of attraction of the globally optimal species configuration now absorbs the entire space of possible initial species configurations (Figure 4).

To examine the evolved partnerships that have enabled these changes in the ecological dynamics, we can display an association matrix, Figure 5 . Figure 5 (left) clearly shows not only that the majority of evolved associations are correct (between species of the same type) but also that they are correctly restricted to partnerships between species in the same sub-systems not across sub-systems. The evolution of partnerships is therefore successful at identifying sub-systems correctly, and identifying correct (single-type) partnerships

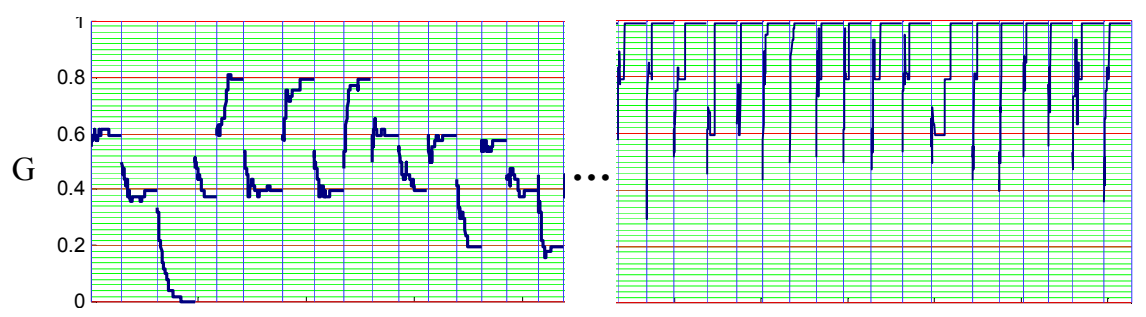

Figure 3: ecosystem dynamics: Left) before associations evolve (initial 10,000 time steps), Right) after associations evolve (around $2.8 \cdot 10^{7}$ time steps). $\mathrm{G}=1 \rightarrow$ globally optimal attractor, $\mathrm{G}=0.2,0.4,0.6,0.8,0.0 \rightarrow$ other local attractors. Vertical lines indicate points at which a new random initial ecological condition is created.

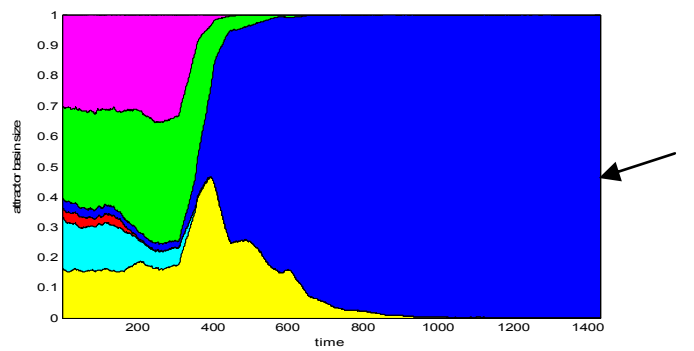

The proportion of initial conditions that reach the globally optimal attractor is intially $1 / 32$ and eventually becomes 1 .

Figure 4: change in size of basins of attraction for different attractor classes over evolutionary time: Shades indicate attractor classes grouped by energy values.
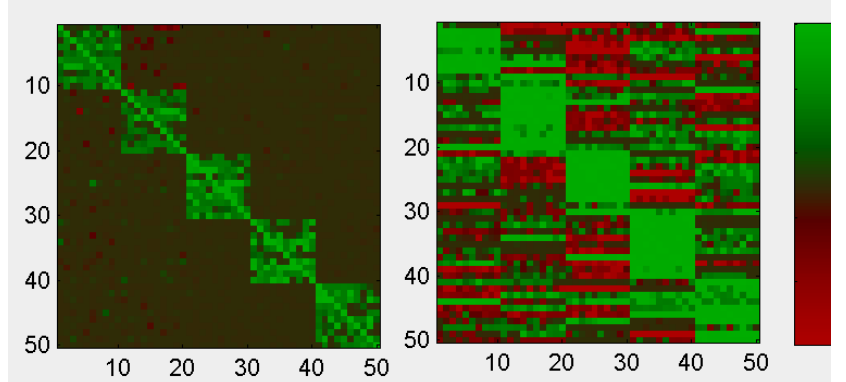

'correct'

associations

no associations

'incorrect'

associations

Figure 5 Evolved associations. Pixel $(i, j)$ depicts the strength and correctness (i.e. 1s with $1 \mathrm{~s}$, and $0 \mathrm{~s}$ with $0 \mathrm{~s}$ ) of the associations between the species $i$ and $j$ (and species $i+N$ and $j+N$ ). Left) associations in the main experiment reveal the modularity of the fitness dependencies defined in the energy function. Right) a control experiment fails to separate modules, see text. 
within those subsystems. Figure 5, right shows that the ability to evolve these correct associations is dependent on the separation of timescales. Specifically, if ecological dynamics are not allowed to settle to a local attractor (by setting $T=1$ in Figure 2), i.e. partnerships evolve in arbitrary ecological contexts, then although they find useful associations within sub-systems, they find incorrect associations between sub-systems.

These results show that individual selection for symbiotic relationships is capable of creating groups that are adaptively significant. After the relationships have evolved, the only attractor of the ecological dynamics is the attractor with the maximal energy. This is surprisingly 'cooperative' since ecological energy corresponds to collective fitness whereas individual selection should just go to the local ESS. The selective pressures that cause individuals to form these groups has two possible components: a) When the ecological context is not yet at an ESS, an individual that brings with it a partner that accelerates approach to the ESS is fitter than one that does not. Thus directional selection on two species promotes symbiosis between them (see (20) for an analogous argument regarding "relational QTLs"). b) When the ecological context is already at an ESS, an individual that brings with it a partner that is also part of the ESS has the same fitness as one that does not (because the species is already present). But an individual that brings a partner that is not part of the ESS will have negative $\Delta \mathrm{e}$ - the partnership is deleterious because it attempts to introduce a species that is selected against in that context. Thus stabilising selection on two species also promotes symbiosis between them albeit in a rather subtle manner.

We suggest that the former direct effect is less significant than the latter subtle effect given that the ecosystem spends most of its time at ESSs. This implies that the fommon form of evolved symbioses is to create associations between species that co-occur most often, and suggests that relationship formation in ecosystems will be basically Hebbian $(15,18,21,22)$ - 'species that fire together wire together'. This has the effect of reinforcing the future co-occurrence of species that already co-occur, and enlarges the basin of attraction for those species combinations in the same manner as Hebbian learning forms an associative memory $(18,22)$. Note that the groups that form do not represent an entire $N$-species ESS but only contain 10 species each (Figure 5) as per the interactions in the energy function. These small groups are both sufficient and selectively efficient in the sense that they create $B$ independent competitions between the two sub-ESSs in each subfunction rather than a single competition between all $2^{B}$ complete ESSs $(19,24)$. These small groups form because the co-occurrence of species within each sub-function is more reliable than the co-occurrence of species in different sub-functions. In (13) we provide a model where we assume that relationships form in a manner that reflects species cooccurrence at ESSs and show that this is sufficient to produce the same effects on attractors as those shown here. Using this abstraction we are also able to assess the scalability of the effect and show that it can evolve rare, high-fitness complexes that are unevolvable via non-associative evolution. This suggests a scalable optimisation method for automatic problem decomposition (24), creating algorithmic leverage similar to that demonstrated by (25).

How does individual selection create higher-level selection? Well, from one point of view it doesn't. If we take into account the combined genetic space of characters, both those addressed directly in the energy function and the genetic loci that control partnerships, then all that happens in our model is that natural selection finds a local attractor in this space. It is only when we pretend not to know about the evolved partnerships, and examine the attractors in the energy function alone, that we see group selection. However, this separation is biologically meaningful and relates to the separation of timescales. That is, the most obvious characteristics of species are those that are under 
direct selection - the ones whose frequencies are affected by selection on short timescales - the ecological population dynamics. But less obvious characteristics are simultaneously under indirect selection - characters that affect co-location of species for example. These change more slowly, over genetic evolution timescales rather than population dynamic timescales (15). When both systems are taken into account, individual selection explains all the observations (if it did not, we would not be satisfied that an evolutionary explanation had been provided). Specifically, partnerships form when group selection is in alignment with individual selection (at ESSs), but in multi-ESS games, these same groupings can cause selection that acts in opposition to (non-associative) individual selection and alter future selective trajectories when individuals are far from that ESS.

Because the indirectly selected characters only have fitness consequences via the directly selected characters their evolution is characterisable by statistics such as cooccurrence of the directly selected characters. This produces systematic consequences on the attractors of directly selected characters - i.e. they enlarge attractors for species combinations reliably found at ESSs. This is equivalent to effecting higher-level selection on these combinations of species. Thus in our opinion, the two types of language - 'higher levels of selection are created' and 'it is all explained by individual selection' - are entirely reconcilable.

Acknowledgements: Chris Buckley, Seth Bullock, Jason Noble, Ivor Simpson, Nicholas Hayes, Mike Streatfield, David Iclanzan, Chrisantha Fernando.

1. J Maynard Smith, E Szathmary (1995) The major transitions in 14. VW Nounburg (1989) A Neural Network Modeled by an Adaptive evalution. Dxfard

Lotka-Volterra System. SIAM Jaurna/ an Applied Mathematics, 49:6. pp. 1779-1792

2. L Margulis (1993) Drigins of species: acquired genomes and individuality. BioSystems 31 (2-3): 121-5

3. DS Wilson (1975) A theory of group selection. PNAS 72(I) 143146

15. FC Paderosa, JF Fontanari (2007) Madel ecosystem with variable interspecies interactions, d. Phys. A: Math. Theor. 40 8723-8738

4. DS Wilsan (1980) The Natura/ Selection of Papulations and I6. NA Moran (2007) Symbiosis as an adaptive process and saurce Cammunities. Benjamin/Cummings of phenotypic complexity. PNAS USA, I0.1073

5. DS Wilsan, LA Dugatkin (1997) Group selection and assartative 17. Stragatz (1994) Non-Linear Dynamics and Chaos, Westview. interactions. The American Naturalist149(2) 336-351 18. RA Watson, C.L. Buckley, Mills, R. (2009) The Effect af Hebbian

6. ST Powers, R Mills, AS Penn, RA Watsan (2009) Sacial niche Learning an Dptimisation in Hapfield Netwarks. Technical construction provides an adaptive explanation far new levels of Report, ECS, University of Sauthampton individuality (ABSTRACT) Submitted to Levels of Selection and 19. RA Watsan, T Jansen (2007) A Building-Black Rayal Road Where Individuality in Evolution Workshop, ECAL 2009. Crossover is Provably Essential. GEECD2007. pp. 1452-1459.

7. ST Powers and RA Watson (2009) Evolution of Individual Group 20. GP Wagner, M Pavlicev, JM Cheverud (2007) The road to Size Preference can Increase Group-level Selection and modularity. Nature Reviews Genetics 8: 921-931 Couperation. To appear ECAL 2009.

8. ST Powers, AS Penn, RA Watson (2007) Individual Selection far Cooperative Group Formation. ECAL 2007. pp. 585-594.

21. RA Watson, R Mills, CL Buckley, N Palmius, ST Pawers, AS Penn (2009b) Hebbian Learning in Ecosystems: Species that fire together wire together (ABSTRACT). To appear ECAL 2009.

9. J Snowdan, ST Powers, RA Watson (2009) Maderate cantact 22. RA Watson, C.L. Buckley, Mills, R. (in prep) Implicit Hebbian between sub-populations promates evolved assartativity Learning and Distributed Dptimisatian in Camplex Adaptive enabling group selection. To appear ECAL 2009. Systems.

10. J Maynard Smith (1982) Evalution and the Theory of Games. 23. RA Watson, JB Pollack (2005) Modular Interdependency in Cambridge

Complex Dynamical Systems. Artificia/Life, II (4). pp. 445-457.

II. R Axelrod (1997) The Complexity af Caoperation. Princeton

12. WD Hamilton (1964) The Genetical Evolution of Social Behaviour I and II, J. Theor. Biol. v7, pp I-52.

13. R Mills, RA Watson (2009) Symbiosis Enables the Evalution of Rare Complexes in Structured Environments. To арреar ECAL 2009

24. R Mills, RA Watson (2009) A symbiosis Algorithm for automatic problem decomposition. In prep.

25. D Iclanzan, D Dumitrescu (2007) Dvercaming hierarchical difficulty by hill-climbing the building black structure. GEССТ 2007. pp 1256 - 1263 